\title{
Postoperative non-steroidal anti-inflammatory drug use and oncological outcomes of rectal cancer
}

\author{
O. Grahn (ID ${ }^{1, *}$, M. Lundin ${ }^{1,2}$, M.-L. Lydrup ${ }^{3}$, E. Angenete (ID ${ }^{4,5}$ and M. Rutegård ${ }^{1,6}$ \\ ${ }^{1}$ Department of Surgical and Perioperative Sciences, Surgery, Umeå University, Umeå, Sweden \\ ${ }^{2}$ Department of Statistics, Umeå School of Business, Economics and Statistics, Umeå University, Umeå, Sweden \\ ${ }^{3}$ Department of Surgery, Skåne University Hospital, Malmö, Lund University, Lund, Sweden \\ ${ }^{4}$ Department of Surgery, Institute of Clinical Sciences, Scandinavian Surgical Outcomes Research Group, Sahlgrenska Academy at University of Gothenburg, \\ Gothenburg, Sweden \\ ${ }^{5}$ Region Västra Götaland, Department of Surgery, Sahlgrenska University Hospital, Gothenburg, Sweden \\ ${ }^{6}$ Wallenberg Centre for Molecular Medicine, Umeå University, Umeå, Sweden \\ *Correspondence to: Department of Surgical and Perioperative Sciences, Umeå University, SE-901 85 Umeå, Sweden (e-mail: oskar.grahn@umu.se)
}

\begin{abstract}
Background: Non-steroidal anti-inflammatory drugs (NSAIDs) are known to suppress the inflammatory response after surgery and are often used for pain control. This study aimed to investigate NSAID use after radical surgical resection for rectal cancer and long-term oncological outcomes.

Methods: A cohort of patients who underwent anterior resection for rectal cancer between 2007 and 2013 in 15 hospitals in Sweden was investigated retrospectively. Data were obtained from the Swedish Colorectal Cancer Registry and medical records; follow-up was undertaken until July 2019. Patients who received NSAID treatment for at least 2 days after surgery were compared with controls who did not, and the primary outcome was recurrence-free survival. Cox regression modelling with confounder adjustment, propensity score matching, and an instrumental variables approach were used; missing data were handled by multiple imputation.

Results: The cohort included 1341 patients, 362 (27.0 per cent) of whom received NSAIDs after operation. In analyses using conventional regression and propensity score matching, there was no significant association between postoperative NSAID use and recurrence-free survival (adjusted hazard ratio (HR) 1.02, 0.79 to 1.33). The instrumental variables approach, including individual hospital as the instrumental variable and clinicopathological variables as co-variables, suggested a potential improvement in the NSAID group (HR 0.61, 0.38 to 0.99).
\end{abstract}

Conclusion: conventional modelling did not demonstrate an association between postoperative NSAID use and recurrence-free survival in patients with rectal cancer, although an instrumental variables approach suggested a potential benefit.

\section{Introduction}

Non-steroidal anti-inflammatory drugs (NSAIDs) are an integral part of multimodal postoperative analgesia in colorectal surgery, providing pain similar relief to opioid analgesia ${ }^{1}$. NSAIDs have also been proven to influence other outcomes, such as reducing the time for recovery of gastrointestinal function and the amount of opioids used ${ }^{2}$. However, there is ongoing concern that NSAID use might increase the frequency of anastomotic leakage based on observational studies ${ }^{3-5}$, although this was not confirmed by others ${ }^{6,7}$ and might depend on tumour location ${ }^{8,9}$. Recent meta-analyses ${ }^{10,11}$ reported conflicting evidence, highlighting that not all NSAIDs seem to carry an increased risk of anastomotic leakage, and that there is a lack of adequately sized RCTs.

In addition, the inflammatory response after surgery, possibly modified by postoperative NSAIDs, might affect the risk of cancer recurrence and survival ${ }^{12}$. Perioperative administration of
NSAIDs has been associated with improved recurrence-free survival in some cancer types, such as breast cancer, and improved overall survival for others, like hepatocellular carcinomas and head and neck cancers ${ }^{10}$. In particular, two recent retrospective cohort studies ${ }^{13,14}$ have shown a significant association between reduced colorectal cancer recurrence, improved recurrence-free survival and perioperative NSAID use. Adequate trial data are difficult to obtain, as large cohorts and long follow-up are needed to prove the expected small differences between study arms. Furthermore, NSAID use has been evaluated mainly in mixed colorectal cancer cohorts, whereas most other research endeavours differentiate between colonic and rectal cancers, not least because of their differing aetiology and treatment ${ }^{15}$.

This study tested the hypothesis that postoperative NSAID treatment improves recurrence-free survival, compared with standard analgesia without NSAIDs, in a cohort of patients who had anterior resection for rectal cancer. 


\section{Methods}

This retrospective multicentre cohort study included all patients who underwent anterior resection for rectal cancer during 20072013 in 15 hospitals in the Northern, Southern, and Western healthcare regions in Sweden. The patients were identified through the Swedish Colorectal Cancer Registry (SCRCR) ${ }^{16}$, which contains information regarding demographics, surgical procedure, postoperative course, oncological treatment, and pathological assessment. The SCRCR has been validated several times, and has almost complete coverage of patients with rectal cancer in Sweden ${ }^{16-18}$. It is checked regularly against the national cancer registry, which receives input from both clinicians and pathologists. The SCRCR defines rectal cancer as an adenocarcinoma of the large bowel, located in the first $15 \mathrm{~cm}$ from the anal verge, as assessed by rigid sigmoidoscopy. Anterior resection was performed according to the principles of total mesorectal excision ${ }^{19}$, which was introduced nationally in the $1990 \mathrm{~s}^{16}$. However, the level of inferior mesenteric artery ligation, splenic flexure mobilization, and the use of minimally invasive surgery differed between study centres and individual surgeons.

The same patient cohort was described in a previous study ${ }^{8}$, with anastomotic leakage as the primary endpoint. Patients with stage IV rectal cancer, all patients who did not undergo radical resection (macroscopically or microscopically), and those who had a palliative resection were excluded from the present analysis. The regional ethical review boards at Umeå University and Gothenburg University approved the protocol (2012-293-31M and 2019-02238, and 647-14).

\section{Data collection and study definitions}

Clinical and demographic variables were collected from the SCRCR, and a chart review was conducted to collect data on smoking status, co-morbidities, and the extent of mesorectal excision $^{8}$. Data on anastomotic leakage within 90 days were gathered from the medical records. Anastomotic leakage was defined and graded according to the International Study Group of Rectal Cancer classification ${ }^{20}$. Patients with anastomotic leakage before the third postoperative day were excluded ${ }^{8}$, as early leaks were considered to be related to technical issues ${ }^{21}$

Data on the study exposure (treatment with any NSAID for at least 2 days within the first week after surgery) were collected from the medical records. Type of NSAID, duration of use until hospital discharge, first postoperative day of use, and total dose were recorded. A dose was only registered if recorded as taken by the patient, as indicated in the medical charts. The primary outcome was recurrence-free survival. Follow-up data from the Colorectal Cancer Registry were collected as at 11 July 2019, including dates of detection of locoregional and distant recurrence, as well as survival data. Patients who emigrated were censored. For those who died, the interval between operation until death was calculated. Secondary outcomes included locoregional and distant recurrence, and overall survival.

\section{Statistical analysis}

Demographic and clinical variables in the no-NSAID and NSAID groups were tabulated, with proportions shown for categorical variables and median (i.q.r.) values for continuous variables. Smoking was not included as data were missing for more than half of the cohort. To visualize survival according to NSAID treatment, Kaplan-Meier curves were plotted and compared using the log rank test.
To test the study hypothesis, Cox regression modelling for the impact of NSAID use on recurrence-free survival was undertaken. Co-variables were selected a priori with the aid of a causal diagram $^{22}$, showing assumptions about causal relations between pertinent variables (Fig. S1). A minimally sufficient set of variables was used to adjust for confounding: age, sex (M or F), ASA grade (I, II or III-IV), BMI (less than 20, 20-25, more than 25 to 30, or over $30 \mathrm{~kg} / \mathrm{m}^{2}$ ), diverting stoma (no or yes), cTNM stage (I, II or III), neoadjuvant therapy (none, radiotherapy or chemoradiotherapy), extent of mesorectal excision (total or partial), tumour height above anal verge, intraoperative blood loss, year of surgery, and individual hospital. Continuous variables were modelled with splines.

Four different sensitivity analyses for the main analysis were performed. Early postoperative death within 90 days was excluded, separate analyses for selective and non-selective NSAIDs were undertaken, as well as stratification for symptomatic leakage within 90 days. In addition, the duration of NSAID administration was used as the exposure (as a continuous as well as a categorical variable) in an attempt to model a dose-response relationship.

Propensity score matching was conducted, using the same covariables as in the regression modelling as matching variables. Propensity scores, that is the conditional probability of receiving NSAID treatment given the values for the co-variables, were estimated in a logistic lasso regression analysis; each patient who had NSAID treatment was matched with one who did not in a one-to-one nearest-neighbour procedure without replacement, minimizing the average imbalance of co-variables between the two groups (Table S1). Thereafter, a Cox regression analysis was performed with the matched sample, with NSAID exposure as the only co-variable to estimate its effect.

An instrumental variables approach was used to assess the effect of NSAIDs on recurrence-free survival. The hospital identifier was used as the instrumental variable in a two-stage residual inclusion procedure, in which the instrumental variable itself was assumed to be associated with the outcome only through treatment. In the first stage, treatment status was regressed on the instrumental variable and the co-variables using ordinary least squares regression. In the second stage, treatment status, covariables, and the residuals from the first-stage analysis were used as predictors in a Cox regression analysis to assess the effect of NSAID use. The set of co-variables other than the instrumental variable itself was the same as for the main Cox regression analysis (see above). Finally, the first Cox regression modelling approach described was used to estimate the secondary outcomes (local and distant recurrence, and overall survival) (Fig. S2).

In all analyses, multiple imputation by chained equations was used to handle missing data. Results from the analyses of imputed data were presented as main results, whereas completecase analyses were undertaken to assess bias introduced by missingness (Table S2). As patients operated at different hospitals could not be treated as independent observations, clustering within hospitals was considered in all analyses.

All statistical tests were two-tailed and the significance level was set at 5 per cent. Stata ${ }^{\circledR}$ version 15.1 (StataCorp, Houston, Texas, USA) and R 3.6.2 (R Foundation for Statistical Computing, Vienna, Austria) were used for statistical analyses.

\section{Results}

A total of 1495 patients who underwent anterior resection and had data regarding NSAID exposure were identified from the 
previous cohort. Some 154 patients were excluded from the present study, with 1341 remaining for analysis (Fig. 1). Of the 154 excluded, 116 had disseminated disease at the time of the operation, 32 did not have radical resection despite having surgery with curative intent, and six patients underwent a planned palliative resection.

Overall, 362 patients (27.0 per cent) were treated with any NSAID for at least 2 days during the first postoperative week, whereas 979 (73.0 per cent) were not. Some 298 patients (82.3 per cent) received non-selective agents and 64 (17.7 per cent) had cyclo-oxygenase (COX) 2-selective agents. Ibuprofen was the most frequently used NSAID, given to 251 of 362 patients (69.3 per cent).

Compared with patients who did not receive NSAIDs, the NSAID group had a lower ASA grade, received neoadjuvant therapy less often, and had TME surgery as well as a diverting stoma more often (Table 1). They had less intraoperative blood loss and also a lower frequency of symptomatic leakage. Moreover, they were slightly younger and less likely to die within 90 days of surgery.

\section{Recurrence-free survival}

The 5-year recurrence-free survival rate was 79.0 and 75.4 per cent in the NSAID and no-NSAID groups respectively. In the unadjusted analysis, the outcome was better in the NSAID group (HR 0.77, 95 per cent c.i. 0.62 to 0.97) (Table 2 and Fig. 2). After adjustment for confounders, however, this effect was not evident (adjusted HR 1.02, 0.79 to 1.33). The result of complete-case analysis was similar (Table S2).

Results were similar in a sensitivity analysis excluding postoperative mortality within 90 days. Stratified analysis according to the presence of symptomatic leakage within 90 days, and analyses of non-selective NSAID and COX-2-selective subgroups also showed no differences between groups (Table S3). In addition, no dose-response association was found when NSAID treatment duration was used as the exposure (Table $\mathrm{S4}$ ).

The results were similar in the propensity score-matched analysis, which showed no significant difference in recurrencefree survival between NSAID and no-NSAID groups (HR 0.89, 0.67 to 1.20) (Fig. 3). On the contrary, with the instrumental variables approach, a positive association between NSAID use and recurrence-free survival was seen (adjusted HR 0.61, 0.38 to 0.99).

\section{Locoregional and distant recurrence, and overall survival}

In the adjusted Cox models, no significant associations were seen between NSAID treatment and locoregional and distant recurrence, or overall survival (Table 2). Point estimates were similar in the complete-case analyses (Table S2).

\section{Discussion}

In this multicentre cohort study of patients who had anterior resection for rectal cancer, an association between postoperative NSAID treatment and recurrence-free survival was not documented, after adjustment for confounding in conventional modelling. However, an instrumental variables approach suggested a potential beneficial effect from NSAIDs, under the strong assumption that individual hospitals did not confer direct effects on survival.

This observational study has several limitations. Residual confounding from misclassification of observed confounders and an influence from unobserved confounders cannot be ruled out. The expertise of individual surgeons and preference regarding NSAID use, which might be confounders, were not considered. However, a previous study ${ }^{23}$ from some of the centres involved in the present analysis suggested that NSAID use is largely driven by postoperative care protocols at each hospital, not by the surgeon; thus, this potential influence is likely to be negated. Although the exposure was assessed carefully using chart review, the outcome data for recurrence were derived only from registry data. The registry has been validated in this respect, with encouraging results, as only 4-5 per cent of recurrence data were found to be erroneous ${ }^{18}$; nevertheless, this misclassification potentially dilutes any association towards the null hypothesis. Overall survival, on the other hand, is not affected as mortality records are virtually always correct in Sweden ${ }^{24}$. Another limitation is the limited sample size, as small benefits with NSAID use in the long term might not be discernible. One of the strengths of the study is the quality of the data, as they were collected from the SCRCR, which has

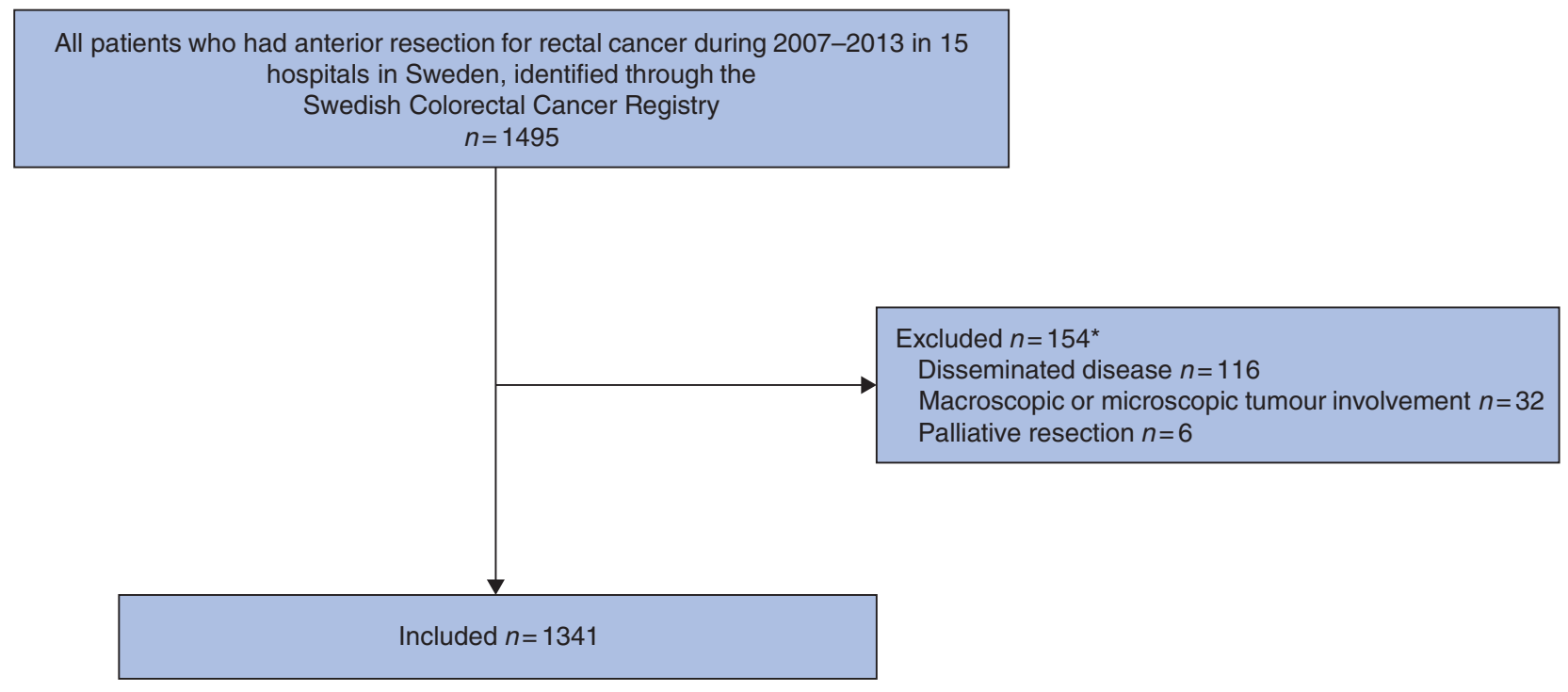


Table 1 Demographic and clinical data for 1341 patients who had anterior resection for rectal cancer during 2007-2013 in Sweden, stratified by any non-steroidal anti-inflammatory drug use for at least 2 days in the first postoperative week

\begin{tabular}{|c|c|c|c|}
\hline & Pos & use & Missing \\
\hline & $\begin{array}{c}\text { No } \\
(n=979)\end{array}$ & $\begin{array}{c}\text { Yes } \\
(n=362)\end{array}$ & \\
\hline Age (years)* & $68(60-74)$ & $65(57-70)$ & $0(0)$ \\
\hline Sex & & & $0(0)$ \\
\hline M & $575(58.7)$ & $195(53.9)$ & \\
\hline F & $404(41.3)$ & $167(46.1)$ & \\
\hline ASA fitness grade & & & $31(2.3)$ \\
\hline I & $228(23.3)$ & $120(33.1)$ & \\
\hline II & $587(60.0)$ & $205(56.6)$ & \\
\hline III & $137(14.0)$ & $33(9.1)$ & \\
\hline BMI $\left(\mathrm{kg} / \mathrm{m}^{2}\right)$ & & & $139(10.4$ \\
\hline$<20$ & $38(3.9)$ & $15(4.1)$ & \\
\hline $20-25$ & $347(35.4)$ & $138(38.1)$ & \\
\hline$>25-30$ & $352(36.0)$ & $156(43.1)$ & \\
\hline$>30$ & $115(11.7)$ & $41(11.3)^{\prime}$ & \\
\hline Neoadjuvant therapy & & & $0(0)$ \\
\hline None & $423(43.2)$ & $132(36.5)$ & \\
\hline Radiotherapy & $444(45.4)$ & $184(50.8)$ & \\
\hline Chemoradiotherapy & $112(11.4)$ & $46(12.7)$ & \\
\hline Surgical approach & & & $12(0.9)$ \\
\hline Open surgery & $805(82.2)$ & $356(98.3)$ & \\
\hline Laparoscopy, not converted & $121(12.4)$ & $1(0.3)$ & \\
\hline Laparoscopy, converted & $46(4.7)$ & $0(0)^{\prime}$ & \\
\hline Type of mesorectal excision & & & $0(0)$ \\
\hline Total & $690(70.5)$ & $274(75.7)$ & \\
\hline Partial & $289(29.5)$ & $88(24.3)$ & \\
\hline Duration of operation (min)* & $253(200-317.5)$ & $261.5(209.5-323.5)$ & $73(5.4)$ \\
\hline Intraoperative blood loss (ml)* & $400(200-700)$ & $300(200-550)$ & $69(5.2)$ \\
\hline Diverting stoma & & & $3(0.2)^{\prime}$ \\
\hline No & $198(20.2)$ & $41(11.3)$ & \\
\hline Yes & $779(79.6)$ & $320(88.4)$ & \\
\hline Anastomotic leakage & & & $0(0)$ \\
\hline No & $837(85.5)$ & $324(89.5)$ & \\
\hline Yes & $142(14.5)$ & $38(10.5)$ & \\
\hline Postoperative mortality $\leq 90$ days & & & $0(0)$ \\
\hline No & $958(97.9)$ & $361(99.7)$ & \\
\hline Yes & $21(2.1)$ & $1(0.3)$ & \\
\hline Tumour height above anal verge $(\mathrm{cm})^{*}$ & $10(9-12)$ & $10(8-12)$ & $\begin{array}{c}10(0.7) \\
288(21.5\end{array}$ \\
\hline $\begin{array}{l}\text { CTNM stage } \\
\text { I }\end{array}$ & $210(21.5)$ & & $288(21.5$ \\
\hline I & $210(21.5)$ & $82(22.7)$ & \\
\hline II & $245(25.0)$ & $86(23.8)$ & \\
\hline III & $314(32.1)$ & $116(32.0)$ & \\
\hline pTNM stage & & & $51(3.8)$ \\
\hline I & $271(27.7)$ & $99(27.4)$ & \\
\hline II & $320(32.7)$ & $95(26.2)$ & \\
\hline III & $343(35.0)$ & $153(42.3)$ & \\
\hline Complete response & $6(0.6)$ & $3(0.8)$ & \\
\hline No. of lymph nodes examined* & $17(13-23)$ & $17(12-23)$ & $11(0.8)$ \\
\hline No. of metastatic lymph nodes* & $0(0-1)$ & $0(0-2)$ & $19(1.4)$ \\
\hline Annual hospital volume (procedures/year)* & $16.1(11-31.9)$ & $14.3(11-31.9)$ & $0(0)$ \\
\hline Year of surgery & $2010(2008-2012)$ & $2010(2009-2011)$ & $0(0)$ \\
\hline
\end{tabular}

Values in parentheses are percentages unless indicated otherwise; *values are median (i.q.r.). NSAID, non-steroidal anti-inflammatory drug.

Table 2 Long-term outcomes for 1341 patients who had with anterior resection for rectal cancer during 2007-2013 in Sweden, of whom 362 received any non-steroidal anti-inflammatory drug use for at least 2 days in the first postoperative week

\begin{tabular}{lccc}
\hline & No. of events & Unadjusted hazard ratio & Adjusted hazard ratio* \\
\hline Recurrence-free survival & 439 & $0.77(0.62,0.97)$ & $1.02(0.79,1.33)$ \\
Locoregional recurrence & 29 & $0.30(0.09,0.99)$ & $0.36(0.10,1.25)$ \\
Distant recurrence & 201 & $0.98(0.72,1.33)$ & $1.05(0.74,1.49)$ \\
Overall survival & 365 & $0.67(0.52,0.87)$ & $0.94(0.70,1.25)$ \\
\hline
\end{tabular}

*Values in parentheses are 95 per cent confidence intervals. With adjustment for ASA grade, age, BMI, intraoperative blood loss, diverting stoma, hospital, neoadjuvant therapy, sex, extent of mesorectal excision, tumour height, year of surgery, and clinical tumour stage. Cox regression modelling was used to derive hazard ratios; multivariable analyses were conducted with a mixed model using multiple imputation to handle missing data 


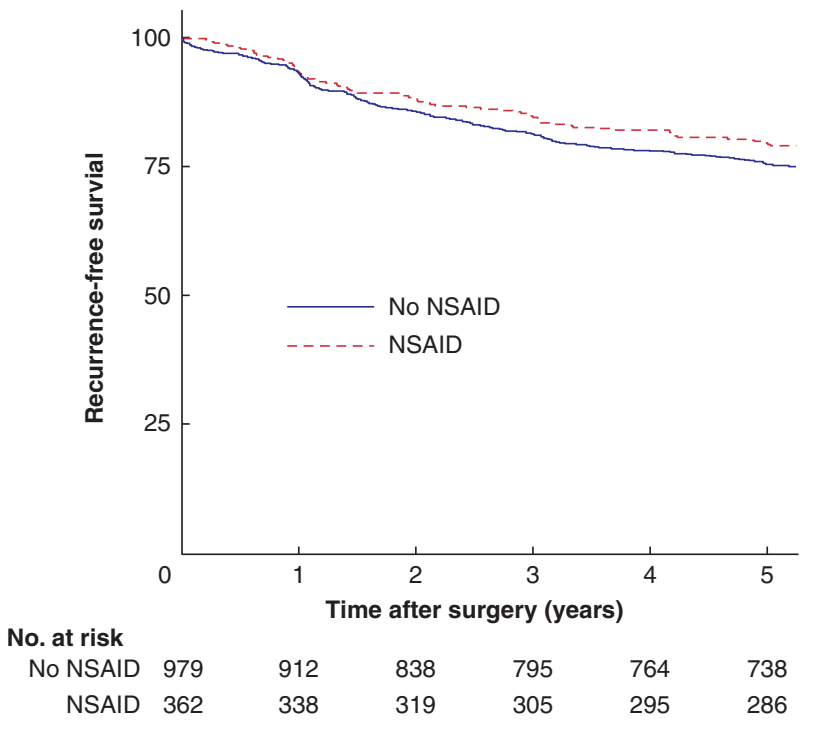

Fig. 2 Unadjusted Kaplan-Meier analysis of recurrence-free survival in 1341 patients operated with anterior resection for rectal cancer, stratified by non-steroidal anti-inflammatory drug use for at least 2 days in the first postoperative week

NSAID, non-steroidal anti-inflammatory drug. $P=0.025$ (log rank test).

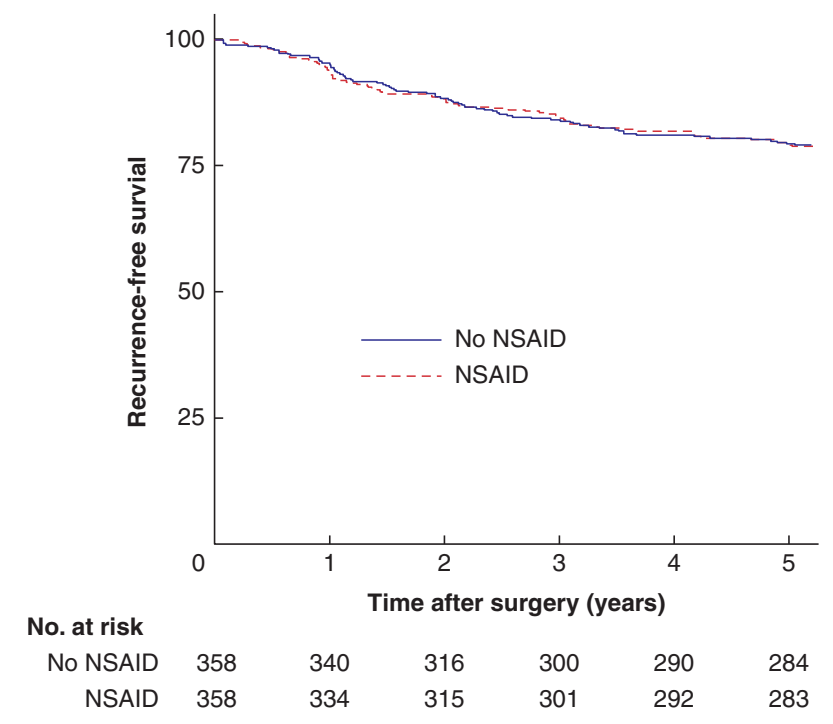

Fig. 3 Kaplan-Meier analysis of recurrence-free survival in propensity score-matched cohort, stratified by non-steroidal anti-inflammatory drug use for at least 2 days in the first postoperative week

NSAID, non-steroidal anti-inflammatory drug. $P=0.690$ (log rank test).

been validated with good results ${ }^{16-18}$, and also supplemented with information from the medical records.

The instrumental variables modelling was done in an attempt to evade confounding issues, but it relies on a strong assumption that any effect of individual hospital (the instrument) on the outcome is directed only through the exposure, NSAID treatment; this weakens this secondary analysis considerably, and the results should be interpreted cautiously. For instance, case volume differed substantially between hospitals (range 17-250 resections during the study period), potentially inducing an effect outside of the exposure path.

Although distributed unevenly between the groups, use of laparoscopy was not included in the confounding set as minimally invasive surgery has not been shown to influence oncological or survival outcomes in multiple trials ${ }^{25}$. However, the open approach has been shown to increase postoperative inflammation $^{26}$, potentially constituting a target for NSAIDs. This motivated a post hoc analysis in which laparoscopy was also included as a co-variable; however, estimates and confidence intervals were virtually unchanged compared with those in the primary Cox regression analysis (data not shown).

A recent publication ${ }^{13}$, using the same definition for exposure, suggested a reduction in cancer recurrence with the use of NSAIDs; in a subgroup analysis, this association was detected in ibuprofen but not diclofenac users. However, disease-free and overall survival were not related to NSAID use and, in an analysis restricting to elective procedures, statistical significance was not reached in terms of recurrence. That study had a larger cohort of 2308 patients, and had greater statistical power than the present analysis, which could in part explain why the results differ. On the other hand, spurious positive findings could also have arisen from multiple testing. Moreover, the present cohort consisted only of elective anterior resections for rectal cancer, potentially leading to differences compared with a heterogenous colorectal cancer cohort, including emergency surgery.

In another new study ${ }^{14}$, perioperative NSAID use was associated with improved survival in patients with rectal cancer. However, such a benefit could be shown only in multivariable analyses stratified for inflammation measured before surgery (in that study defined as a high platelet-to-lymphocyte ratio); the NSAID group with a more pronounced inflammatory state had significantly better disease-free survival. The inflammatory response is usually increased in the acute setting, possibly explaining why the aforementioned cohort study ${ }^{13}$ could only report positive findings when emergency procedures were included. Unfortunately, inflammation status was not determined in the present study. In the study of Huang and colleagues ${ }^{14}$, the recurrence rate in the NSAID and no-NSAID groups was 16 and 29 per cent respectively. Assuming such a large difference, a statistical power of 90 per cent could be achieved by enrolling 560 patients.

Nevertheless, the results above indicate that NSAIDs should be studied further regarding long-term outcomes after colorectal cancer surgery, perhaps with a focus on patients with a preoperative inflammatory condition. These studies should ideally consider different tumour locations in the colorectum as well as the inflammatory state, including whether the procedure itself is an elective or emergency operation.

Surgical trauma and inflammation are known to increase the risk of tumour cell dissemination; proinflammatory cytokines can stimulate adhesion of circulating cancer cells, and catecholamines and prostaglandins can promote the invasion of circulating cancer cells ${ }^{27}$. NSAIDs in the postoperative setting, with their anti-inflammatory actions, may counteract minimal residual disease regarding both persistence and invasiveness. These agents have been shown to inhibit tumour-associated inflammation and metastatic disease in experimental models of cancer, and perioperative NSAIDs are also associated with improved outcomes in humans for some other forms of cancer such as hepatocellular carcinoma ${ }^{28}$.

This study found no association between use of postoperative NSAIDs and recurrence-free survival, local or distant recurrence, or overall survival. It would be of value to study different types of NSAID and different locations in the colorectum. Moreover, the preoperative inflammatory state might be an important effect modifier for NSAID use in relation to recurrence. At present, the 
evidence is sparse and conflicting, and the question remains whether or not NSAID treatment is protective.

\section{Acknowledgements}

The study received funding from the Knut and Alice Wallenberg Foundation, and the Cancer Research Foundation in Northern Sweden.

Disclosure. The authors declare no conflict of interest.

\section{Supplementary material}

Supplementary material is available at BJS Open online.

\section{References}

1. Gustafsson UO, Scott MJ, Hubner M, Nygren J, Demartines N, Francis $\mathrm{N}$ et al. Guidelines for perioperative care in elective colorectal surgery: enhanced recovery after surgery (ERAS ${ }^{\circledR}$ ) Society Recommendations: 2018. World J Surg 2019;43:659-695

2. Chapman SJ, Garner JJ, Drake TM, Aldaffaa M, Jayne DG. Systematic review and meta-analysis of nonsteroidal antiinflammatory drugs to improve gi recovery after colorectal surgery. Dis Colon Rectum 2019;62:248-256

3. Hakkarainen TW, Steele SR, Bastaworous A, Dellinger EP, Farrokhi E, Farjah F et al. Nonsteroidal anti-inflammatory drugs and the risk for anastomotic failure: a report from Washington State's Surgical Care and Outcomes Assessment Program (SCOAP). JAMA Surg 2015;150:223-228

4. Bakker N, Deelder JD, Richir MC, Cakir H, Doodeman HJ, Schreurs WH et al. Risk of anastomotic leakage with nonsteroidal anti-inflammatory drugs within an enhanced recovery program. J Gastrointest Surg 2016;20:776-782

5. Klein M, Gögenur I, Rosenberg J. Postoperative use of nonsteroidal anti-inflammatory drugs in patients with anastomotic leakage requiring reoperation after colorectal resection: cohort study based on prospective data. BMJ 2012;345:e6166

6. Collaborative STARSurg. Safety of nonsteroidal antiinflammatory drugs in major gastrointestinal surgery: a prospective, multicenter cohort study. World J Surg 2017;41:47-55

7. Collaborative EuroSurg. Safety and efficacy of non-steroidal anti-inflammatory drugs to reduce ileus after colorectal surgery. Br J Surg 2020;107:e161-e169

8. Kverneng Hultberg D, Angenete E, Lydrup ML, Rutegard J, Matthiessen P, Rutegard M. Nonsteroidal anti-inflammatory drugs and the risk of anastomotic leakage after anterior resection for rectal cancer. Eur J Surg Oncol 2017;43:1908-1914

9. Yauw ST, Lomme RM, van der Vijver RJ, Hendriks T, van Laarhoven KJ, van Goor H. Diclofenac causes anastomotic leakage in the proximal colon but not in the distal colon of the rat. Am J Surg 2015;210:382-388

10. Cata JP, Guerra CE, Chang GJ, Gottumukkala V, Joshi GP. Nonsteroidal anti-inflammatory drugs in the oncological surgical population: beneficial or harmful? A systematic review of the literature. Br J Anaesth 2017;119:750-764

11. Modasi A, Pace D, Godwin M, Smith C, Curtis B. NSAID administration post colorectal surgery increases anastomotic leak rate: systematic review/meta-analysis. Surg Endosc 2019;33:879-885
12. Behrenbruch C, Shembrey C, Paquet-Fifield S, Molck C, Cho HJ, Michael $\mathrm{M}$ et al. Surgical stress response and promotion of metastasis in colorectal cancer: a complex and heterogeneous process. Clin Exp Metastasis 2018;35:333-345

13. Schack A, Fransgaard T, Klein MF, Gogenur I. Perioperative use of nonsteroidal anti-inflammatory drugs decreases the risk of recurrence of cancer after colorectal resection: a cohort study based on prospective data. Ann Surg Oncol 2019;26:3826-3837

14. Huang Z, Wang X, Zou Q, Zhuang Z, Xie Y, Cai D et al. High platelet-to-lymphocyte ratio predicts improved survival outcome for perioperative NSAID use in patients with rectal cancer. Int J Colorectal Dis 2020;35:695-704

15. Li FY, Lai MD. Colorectal cancer, one entity or three. J Zhejiang Uniu Sci B 2009;10:219-229

16. Pahlman L, Bohe M, Cedermark B, Dahlberg M, Lindmark G, Sjodahl R et al. The Swedish rectal cancer registry. Br J Surg 2007; 94:1285-1292

17. Moberger P, Skoldberg F, Birgisson H. Evaluation of the Swedish Colorectal Cancer Registry: an overview of completeness, timeliness, comparability and validity. Acta Oncol 2018;57:1611-1621

18. Jorgren F, Johansson R, Damber L, Lindmark G. Validity of the Swedish Rectal Cancer Registry for patients treated with major abdominal surgery between 1995 and 1997. Acta Oncol 2013;52: 1707-1714

19. Heald RJ, Husband EM, Ryall RD. The mesorectum in rectal cancer surgery-the clue to pelvic recurrence? Br J Surg 1982;69: 613-616

20. Rahbari NN, Weitz J, Hohenberger W, Heald RJ, Moran B, Ulrich A et al. Definition and grading of anastomotic leakage following anterior resection of the rectum: a proposal by the International Study Group of Rectal Cancer. Surgery 2010;147:339-351

21. Thompson SK, Chang EY, Jobe BA. Clinical review: healing in gastrointestinal anastomoses, Part I. Microsurgery 2006;26: 131-136

22. Textor J, van der Zander B, Gilthorpe MS, Liśkiewicz M, Ellison GT. Robust causal inference using directed acyclic graphs: the R package 'dagitty'. Int J Epidemiol 2017;45:1887-1894

23. Rutegård M, Westermark S, Kverneng Hultberg D, Haapamäki M, Matthiessen P, Rutegård J. Non-steroidal anti-inflammatory drug use and risk of anastomotic leakage after anterior resection: a protocol-based study. Digest Surg 2016;33:129-135

24. Brooke HL, Talbäck M, Hörnblad J, Johansson LA, Ludvigsson JF, Druid H et al. The Swedish cause of death register. Eur J Epidemiol 2017;32:765-773

25. Nienhüser H, Heger P, Schmitz R, Kulu Y, Diener MK, Klose J et al. Short- and long-term oncological outcome after rectal cancer surgery: a systematic review and meta-analysis comparing open versus laparoscopic rectal cancer surgery. J Gastrointest Surg 2018;22:1418-1433

26. Veenhof AA, Sietses C, von Blomberg BM, van Hoogstraten IM, vd Pas MH, Meijerink WJ et al. The surgical stress response and postoperative immune function after laparoscopic or conventional total mesorectal excision in rectal cancer: a randomized trial. Int J Colorectal Dis 2011;26:53-59

27. Tohme S, Simmons RL, Tsung A. Surgery for cancer: a trigger for metastases. Cancer Res 2017;77:1548-1552

28. Hiller JG, Perry NJ, Poulogiannis G, Riedel B, Sloan EK. Perioperative events influence cancer recurrence risk after surgery. Nat Reu Clin Oncol 2018;15:205-218 